\title{
Fatal arterial hemorrhage after microwave ablation of multiple liver metastases: The lessons learned
}

\author{
ADAM HATZIDAKIS ${ }^{1,2}$, NIKOLAOS ZERVAKIS ${ }^{3}$, MILTIADIS KROKIDIS $^{4 *}$ \\ ${ }^{1}$ Department of Radiology, Heraklion University Hospital, Heraklion, Greece \\ ${ }^{2}$ Department of Radiology, Creta Interclinic Hospital, Heraklion, Greece \\ ${ }^{3}$ Department of Surgery, Creta Interclinic Hospital, Heraklion, Greece \\ ${ }^{4}$ Department of Radiology, Cambridge University Hospitals NHS Trust, Cambridge, UK \\ *Corresponding author: Miltiadis Krokidis MD, PhD, EBIR, Consultant Vascular and Interventional Radiologist; \\ Department of Radiology, Cambridge University Hospitals NHS Trust; Hills Road, CB2 0QQ, Cambridge, United Kingdom; \\ Phone: +44 1223 348920; Fax: +44 1223 217847; E-mail: mkrokidis@hotmail.com
}

(Received: April 28, 2013; Revised manuscript received: June 24, 2013; Accepted: June 25, 2013)

\begin{abstract}
We report the case of a 71-year-old female patient with previous history of rectal cancer. The primary cancer was resected and three cycles of chemotherapy were completed. Excision of metastatic lesions was also performed from the left liver lobe. Due to the presence of new liver lesions on the right lobe, intra-operative microwave ablation was decided. Three hours post ablation the patient bled massively and angiography revealed extravasation from a peripheral branch of the right hepatic artery. Successful embolization was performed; however, the patient died 5 days later on liver failure. We would like to present the case and discuss the technical options and the key points to help avoiding similar complications in the future.
\end{abstract}

Keywords: liver metastases, microwave ablation, complications, hemorrhage

\section{Introduction}

Surgical resection of hepatic metastases from colorectal cancer is still considered as the gold standard for disease free long-term survival and a mortality rate of less than 5\% [1]. Patient suitability for resection is not yet welldefined and additional methods, such as neoadjuvant chemotherapy, pre-operative portal vein embolization and 2 -stage resections have increased the number of patients eligible for surgery [1]. Nevertheless, the majority of patients remain without indication for curative surgery. Recently, several interstitial ablative techniques have shown promising results in unresectable liver cancers [2].

Radiofrequency (RFA) and microwave ablation (MWA) of liver metastases are two out of those techniques, performed under imaging guidance either percutaneously or intra-operatively. Both methods are considered as safe and effective, with very low morbid- ity and mortality rates [3-7]. Patient death caused by massive arterial bleeding after ablation of liver lesions is very rare [6-10]. We present such a case with special attention to the reasons caused this complication and suggestions on how to avoid them in the future.

\section{Case Report}

A 71-year-old female patient with history of rectal cancer was referred to our department from oncology. The patient was operated for the primary rectal cancer four years earlier. Six months later liver metastases were discovered. There was a $0.7-\mathrm{cm}$ lesion in segment VI, a 0.8 - and a $1.3-\mathrm{cm}$ lesion in segment II and a $1.2-\mathrm{cm}$ lesion in segment III. The patient received chemotherapy and partial response was achieved. Excision of the remaining metastatic deposits post chemotherapy was decided and the three lesions were removed from the 


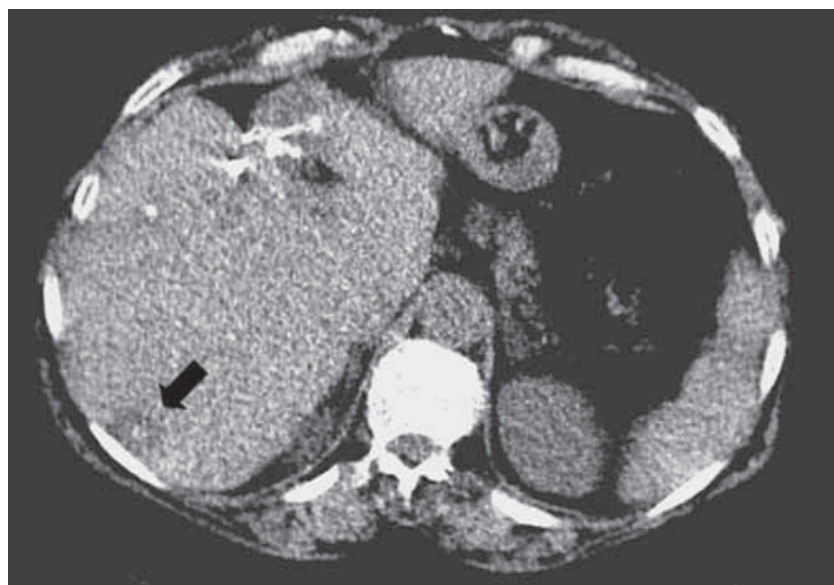

Fig. 1. Computed Tomography scan showing post-surgical changes in the left liver lobe after resection of metastatic lesions. In the right lobe new lesions were found, one of them on this image (arrow)

left lobe (located in segments II and III). Another year later, new lesions were detected and a new chemotherapy regime was administered. However, 5 residual lesions were still detected in the Computed Tomography (CT) scan post chemotherapy (Figs 1 and 2). The patient was considered as a very poor candidate for further hepatectomy or chemotherapy due to deterioration of the liver function and was referred to us in view of ablation of the residual multiple metastatic lesions.

Due to the number and the location of the lesions, intra-operative approach and ablation with microwave was decided. All 5 lesions were located in the right liver lobe. A microwave (MW)-generator (MedWaves, San Diego, USA) was used, with an Avecure 14 G, 23-cmlong needle. Guidance of the probe with ultrasound was performed. A 3.5- $\mathrm{MHz}$ curvilinear probe was used. All the lesions were successfully ablated in the same session.

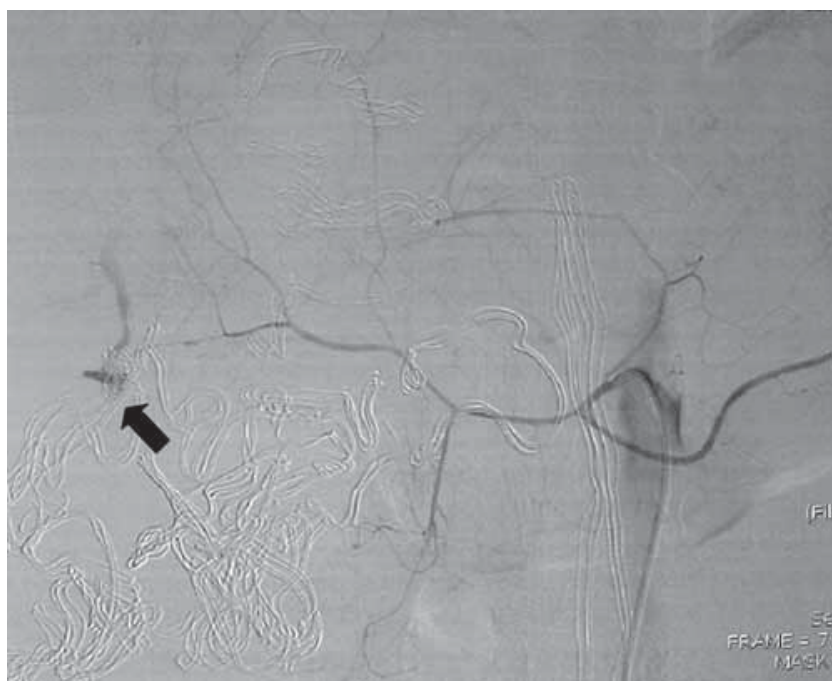

Fig. 3. Selective hepatic arteriography revealing arterial spasm and contrast extravasation from an iatrogenic damaged right hepatic arterial branch (arrow)

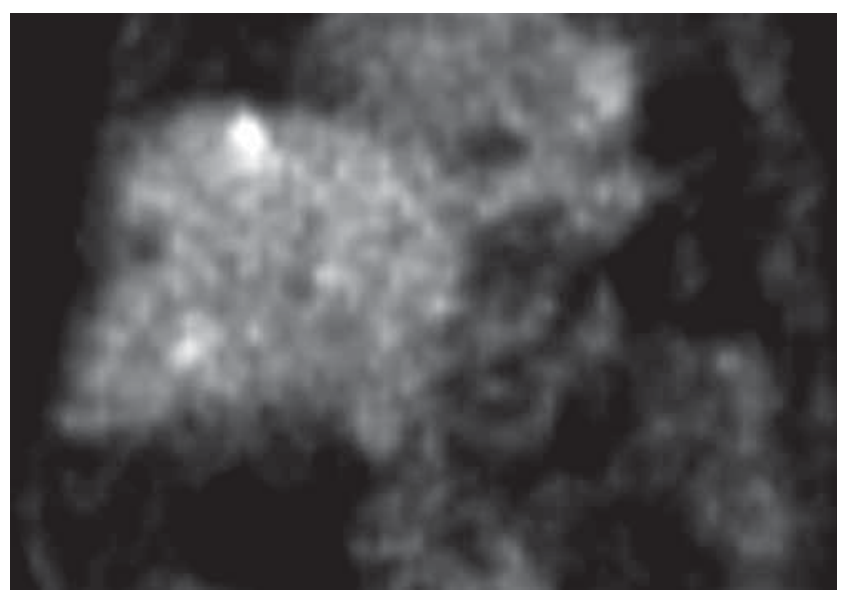

Fig. 2. PET-CT scan showing multiple locations of radioactive enhancement in the right liver lobe, suggesting new meta-
static lesions

The ablation generator produces $45 \mathrm{~W}$ of power at a frequency of $915 \mathrm{MHz}$. Ablation time was $10 \mathrm{~min}$ for each lesion and the total diameter achieved for each lesion was $4 \mathrm{~cm}$. The total procedure time was approximately 3 hours. After the ablation, surgical closure was performed and a surgical drain was positioned in the sub-hepatic space. The patient was transferred to the ward and appeared to be recovering well for the first three hours post procedure. Then, sudden dramatic elevation of the systolic blood pressure was noticed (over $200 \mathrm{mmHg}$ ) with immediate bleeding from the surgical drain indicating hemorrhage from the sub-hepatic space. The patient was immediately transferred to the operating theatre and a new laparotomy was performed. Bleeding from the liver capsule was detected and packing with radio-opaque gauzes followed. The patient was stabilized and transferred to the angio-suite for selective hepatic

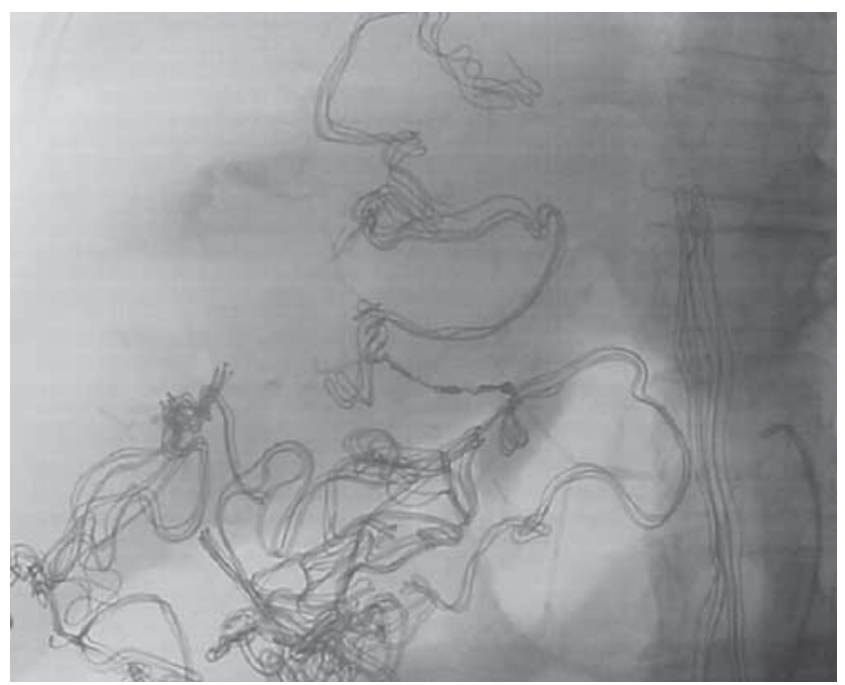

Fig. 4. Post-embolization image with impacted coils before the extravasating branch. Multiple radio-opaque surgical gauzes are seen around the liver capsule 


\section{Hatzidakis et al.}

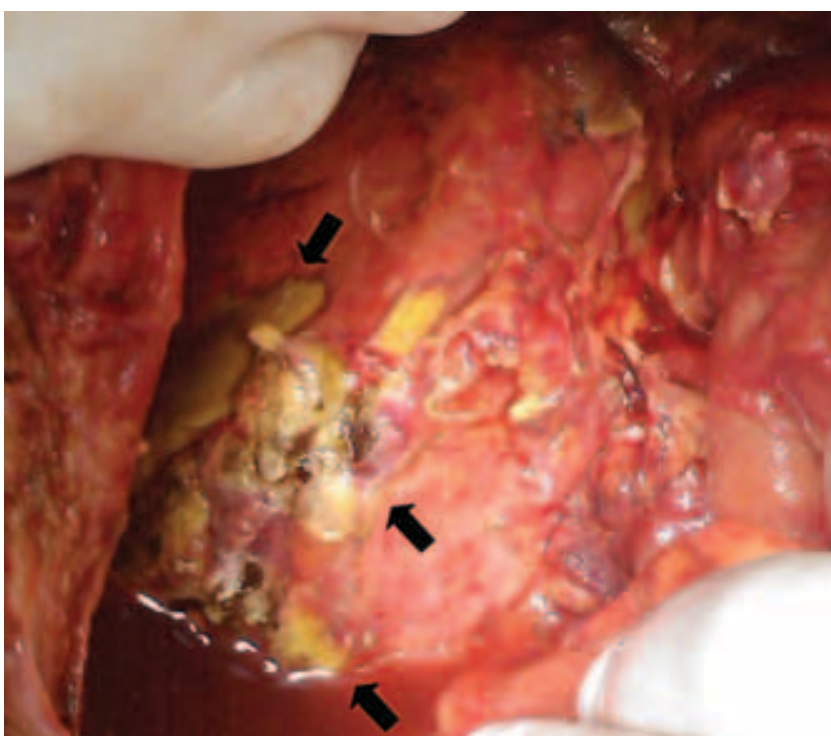

Fig. 5. Intra-abdominal view of the liver after removal of the surgical gauzes, showing extended liver necrosis (arrows)

arteriography. Angiogram revealed diffuse arterial spasm and contrast extravasation from a right hepatic artery branch (Fig. 3), which was subsequently embolized with two metallic 0.018 -inch micro coils, $3 \times 50 \mathrm{~mm}$ and $4 \times$ $120 \mathrm{~mm}$ (BALT, Montmorency, France) (Fig. 4), with satisfactory result.

The patient was transferred in the Intensive Care Unit (ICU) and was transfused further on. Two days later a new laparotomy was performed and after removal of the gauzes, extended blood extravasation was seen from the liver capsule, in form of diffuse subcapsular haematoma. Liver was re-tamponated and three days later the procedure was repeated. This time no bleeding was found, but segmental necrosis was macroscopically seen (Fig. 5). The patient died two days later due to liver failure.

\section{Discussion}

Liver is the second most common site of metastasis from colorectal primary cancers $[3,11]$. The potential cure in such cases may be offered by surgical resection [12]. However, the nature of the disease is such that continuous progression occurs and chemotherapy is not always possible to contain this progression. New lesions usually appear and surgical options are limited in such cases. Looking for less invasive solutions thermal ablation offers an alternative to surgical intervention [11]. Several interstitial ablative techniques have shown promising results in percutaneous treatment of unresectable liver cancers [2-4]. Radiofrequency and microwave ablation of liver metastases are the most established techniques, performed either percutaneously or intra-operatively [5-11]. Advantages of thermal ablative techniques are repeatability, shorter hospital stay and lower morbidity and mortality rates [11]. Especially MWA seems to be more effective due to higher temperatures, larger tumor ablation volumes and faster ablation times [13]. Major complication rate requiring treatment is reported lower than $5 \%$, while procedure-related mortality is considered as relative rare, so that these methods can be characterized as safe [5-11, 14-16].

Livraghi et al. reported major arterial bleeding after liver RFA in just $0.5 \%$ (12 out of 2320 patients) [8]. In 3 patients surgery was necessary and in another one intra-arterial embolization. Only one patient died after massive hemorrhage that was caused by tumor rupture. Actually, this is in our knowledge the only patient death reported from massive hemorrhage after percutaneous radiofrequency ablation until now.

In a meta-analysis of primary and secondary liver malignancies ablated with MW, an ablation associated mortality rate of just $0.002 \%$ was found [5]. In another more recent meta-analysis performed by Bertot et al. in 2011 , the percutaneous performed ablative methods were considered as safe, with a major complication rate of $4.1 \%$ and $4.6 \%$ for RFA and MWA, respectively [6]. The technique-related mortality rate is rather lower, reaching $0.15 \%$ and $0.23 \%$ for RFA and MWA, respectively [6]. In this review of 1,185 cases of MWA, the only deaths reported were due to cardiac or multi-organ failure, while in 9,531 cases of RFA, deaths were found because of colon perforation, peritonitis, liver failure, bile leakage, tumor rupture with massive hemorrhage and peritoneal hemorrhage [6].

Livraghi et al. [7] in another study, reported no mortality and a major complication rate of $2.9 \%$, with two only cases of hemorrhage requiring blood transfusion $(0.3 \%)$ [7]. However, in that study patients with lesions not larger than $5 \mathrm{~cm}$ were included and no more than 4 lesions per session were treated with MVA.

Also when performed in combination with surgical resection, most complications like wound infection, biliary fistula or transient liver failure most likely occurred because of the surgical techniques and less due to the ablation [1]. Martin et al. treated 100 patients with 270 hepatic tumors by combining hepatic resection with MWA [10]. Fifty of them suffered from metastatic liver disease with a mean of 2 lesions (range 1-12). Patients with multiple lesions in different liver segments were ablated under open surgical inspection and ultrasound guidance. No mortality or hemorrhage was found, while major complication was noted once due to a hepatic abscess formation [10].

In a larger series with 1,928 hepatic tumors ablated percutaneously with MW an intrahepatic hemorrhage was reported in one uncooperative patient [9]. Patient required arterial embolization four hours later due to blood pressure decrease but survived. Also two non-ablation related deaths, one from respiratory and cardiac insufficiency and one from multi-organ failure 
were noted. The authors believe that increased number of procedures is a factor associated with higher rate of complications [9].

\section{Decision Making}

In our case, the patient bled from a damaged arterial vessel. This is not a frequent complication because firstly arteries are rarely damaged by direct needle puncture and in the case that an artery is slightly eroded by the thermal ablation of structures it is expected to heal during the coagulation process. Apparently the needle was retrieved quicker than whet it was required in order to obtain the necessary coagulation effect in the damaged vessel. Nevertheless, the lesion was subsequently embolized successfully. However, the embolization effect was of negative impact on an organ with multiple problems and in addition to the extensive coagulation necrosis caused by the MWA of multiple lesions let to fatal liver failure.

A procedure in two or three stages maybe would have been a better option considering the background and the rather poor reserves of the patient. Embolization could not have been avoided and may be potentially necessary after any percutaneous liver case. Needle placement through healthy parenchyma is crucial in order to be able to seal the potential hemorrhage and in case of ablation appropriate time for coagulation is required to avoid seeding and to try and repair any damaged vessel.

\section{Conflict of Interest}

The authors declare no conflict of interest.

\section{References}

1. Tanaka K, Shimada H, Nagano Y, Endo I, Sekido H, Togo S: Outcome after hepatic resection versus combined resection and microwave ablation for multiple bipolar colorectal metastases to the liver. Surgery 139, 263-273 (2006)

2. Erce C, Parks RW: Interstitial ablative techniques for hepatic tumours. Br J Surg 90, 272-289 (2003)
3. Gillams AR, Lees WR: Five-year survival following radiofrequency ablation of small, solitary, hepatic colorectal metastases. J Vasc Intervent Radiol 19, 712-717 (2008)

4. Flanders VL, Gevrais DA: Ablation of liver metastases: Current status. J Vasc Interv Radiol 21, S214-S222 (2010)

5. Ong SL, Gravante G, Metcalfe MS, Strickland AD, Dennison AR, Lloyd DM: Efficacy and safety of microwave ablation for primary and secondary liver malignancies: A systematic review. Eur Gastrenterol Hepatol 21, 599-605 (2009)

6. Bertot LC, Sato M, Tateishi R, Yoshida H, Koike K: Mortality and complication rates of percutaneous ablative techniques for treatment of liver tumors: A systematic review. Eur Radiol 21, 2584-2596 (2003)

7. Livraghi T, Meloni F, Solbiati L, Zanus G; Collaborative Italian Group using AMICA system: Complications of microwave ablation for liver tumors: Results of a multicenter study. Cardiovasc Intervent Radiol 35, 868-874 (2012)

8. Livraghi T, Solbiati L, Meloni MF, Gazelle GS, Halpern EF, Goldberg SN: Treatment of focal liver tumors with percutaneous radiofrequency ablation: Complications encountered in a multicenter study. Radiology 226, 441-451 (2003)

9. Liang P, Wang Y, Yu X, Dong B: Malignant liver tumors: Treatment with percutaneous microwave ablation: Complications among cohort of 1136 patients. Radiology 251, 933-940 (2009)

10. Martin RC, Scoggins CR, McMasters KM: Safety and efficacy of microwave ablation of hepatic tumors: A prospective review of a 5-year experience. Ann Surg Oncol 17, 171-178 (2010)

11. Jones C, Badger SA, Ellis G: The role of microwave ablation in the management of hepatic colorectal metastases. Surgeon 9, 33-37 (2011)

12. Rees M, Tekkis PP, Welsh FK, O'Rourke T, John TG: Evaluation of long-term survival after hepatic resection for metastatic colorectal cancer: A multifactorial model of 929 patients. Ann Surg 247, $125-135$ (2008)

13. Simon CJ, Dupuy DE, Mayo-Smith WW: Microwave ablation: Principles and applications. Radiographics 25, S69-S83 (2005)

14. McWilliams JP, Yamamoto S, Raman SS, Loh CT, Lee EW, Liu DM, Kee ST: Percutaneous ablation of hepatocellular carcinoma: Current status. J Vasc Interv Radiol 21, S204-S213 (2010)

15. Hartmann E, Németh A, Juharosi Gy, Lénárd Zs, Deák PÁ, Kozma V, Nagy P, Gerlei Zs, Fehérvári I, Nemes B, Görög D, Fazakas J, Kóbori L, Doros A: Downstaging of hepatocellular carcinoma with radiofrequency ablation on the Hungarian liver transplantation waiting list - Early results and learned lesson. IMAS 1, 41-45 (2009)

16. Kozma V, Végső Gy, Deák PÁ, Hartmann E, Németh A, Török Sz, Langer R, Doros A: Radiofrequency ablation of an intercalyceal neoplasm in a transplanted kidney using percutaneous nephrostomy for cooling - Safety and early result. IMAS 2, 37-41 (2010) 